\title{
Early expression of requisite developmental growth hormone imprinted cytochromes P450 and dependent transcription factors
}

\author{
Sarmistha Banerjee, Allison M Hayes and Bernard H Shapiro \\ Department of Biomedical Sciences, University of Pennsylvania, Philadelphia, Pennsylvania, USA
}

Correspondence should be addressed to B H Shapiro: shapirob@upenn.edu

\begin{abstract}
The sexually dimorphic expression of cytochromes P450 (CYP) drug metabolizing enzymes

has been reported in all species examined. These sex differences are initially expressed

during puberty and are solely regulated by sex differences in the circulating growth

hormone $(\mathrm{GH})$ profiles. Once established, however, the different male- and female-

dependent CYP isoforms are permanent and immutable, suggesting that adult CYP

expression requires imprinting. Since the hormone that regulates an adult function is

likely the same hormone that imprints the function, we selectively blocked GH secretion in

some newborn male rats while others also received a concurrent physiologic replacement of rat GH. Rats were subsequently challenged, peripubertally, with either a masculine-like episodic GH regimen or the $\mathrm{GH}$ vehicle alone. The results demonstrate that episodic $\mathrm{GH}$ regulation of male-specific CYP2C11 and CYP3A2, as well as female-predominant CYP2C6, are dependent on developmental GH imprinting. Moreover, the induction and/or activation of major components in the signal transduction pathway regulating the expression of the principal CYP2C11 isoform is obligatorily dependent on perinatal GH imprinting without which CYP2C11 and drug metabolism would be permanently and profoundly suppressed. Since there are additional adult metabolic functions also regulated by $\mathrm{GH}$, pediatric drug therapy that is known to disrupt GH secretion could unintentionally impair adult health.
\end{abstract}

Key Words

- CYP

- growth hormone

- imprinting

- P450s

- sexual dimorphism

- transcription factors

\section{Introduction}

Sexual dimorphisms of some dozen or more hormone- and drug-metabolizing constituent cytochromes P450 (CYPs) observed in rats, humans, and many other species examined (1) are defined by two characteristics. (i) Following puberty, males and females express different CYP profiles. In some cases, the isoforms may be sex exclusive as is the case of CYP2C12, only expressed in female rats $(1,2)$. More commonly, isoforms are sex predominant: for example, women express greater levels of CYP3A4 and CYP3A5 $(3,4)$ while in the case of mice, males express greater levels of CYP2D9 (2). (ii) The sexual dimorphisms in CYP expression are determined by sex differences in the circulating growth hormone $(\mathrm{GH})$ profiles in adulthood. More specifically, it is not the amount of circulating GH per se, but rather, the sexually dimorphic ultradian rhythms in plasma $\mathrm{GH}$ that regulate sex-dependent isoforms of CYP. Although there are some variations between species, in general, males secrete $\mathrm{GH}$ in what is referred to as an 'episodic' profile characterized by several daily bursts of hormone separated by lengthy undetectable or barely detectable GH concentrations. In contrast, the adult female GH profile is considered 'continuous' as there are far more secretory bursts, often at lower amplitudes of the hormone separated by briefer interpulse periods, often containing measurable levels of GH. In fact, these sex differences in the circulating $\mathrm{GH}$ profile are solely responsible for the sex difference in

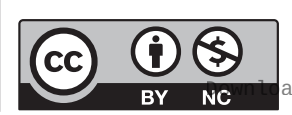

This work is licensed under a Creative Commons Attribution-NonCommercial 4.0 International License. ded from Bioscientifica.com at 04/26/2023 01:06:54PM 
CYP expression and resulting drug metabolism observed during adulthood $(1,5)$.

Most, if not all, sexual dimorphisms are a result of developmental hormone imprinting. Hormonal imprinting refers to a biological process in which the target tissue becomes responsive to the hormone. During the initial exposure, the hormone irreversibly reprograms the development of the affected tissue, likely through epigenetic alterations (6), so as to permanently alter some functional aspect normally responsive to the hormone and often establishing a sexual dimorphism (7). Moreover, the tissue is programmable for only a brief developmental period, after which time the tissue becomes permanently unresponsive to imprinting $(8,9,10)$. Imprinting alone, however, is generally insufficient to ensure the expression of the programmed function. Accordingly, the expression of the affected function requires both imprinting and activation, the latter being a reversible process but required to express the imprinted function. For example, perinatal testosterone or its androgen/estrogen metabolites are required to permanently 'wire' or imprint the male brain to exclusively express masculine sexual behavior. However, the brain has to be stimulated or activated in adulthood by the same hormone to elicit the imprinted male sexual behavior. Both imprinting and activation are required for normal masculine sexual behavior $(9,10,11)$, which explains why perinatally castrated males and intact females, not hormonally imprinted, are unable, regardless of adult hormone treatment, to exhibit normal masculine sexual behavior $(9,11)$.

In this regard, we previously reported that adult male rats and men cannot be induced (regardless of GH treatment) to express the normal female profile of hepatic CYPs $(3,12$, $13,14,15)$ nor can adult female rats or women be induced to fully express the masculine CYP profile $(14,16,17,18)$. If CYP enzymes were not imprinted, then irrespective of sex, the same adult treatment should produce the same CYP expression levels in males and females. Since this is not the case, we had concluded that the sex differences in adult expression profiles of CYPs are permanent and irreversible, a condition demonstrating imprinting. Because an adult tissue/function is responsive to the same hormone by which it was imprinted, we had proposed, for the first time, that like androgens, perinatal GH is a developmental organizer required for the normal expression of adult CYPdependent drug metabolism. In this regard, we reported that adult expression of the principal male-specific CYP2C11 isoform in the rat is completely dependent on imprinting by developmental GH. In the absence of the perinatal hormone, CYP2C11 (comprising $>50 \%$ of the total male hepatic CYP content (19)) is permanently and irreversibly unresponsive to the inductive effects of the masculine episodic GH profile $(6,20,21)$.

In our previous reports, we used primary hepatocyte cultures derived from adult male rats to demonstrate the permanent effects of developmental GH imprinting on CYP2C11 expression $(6,21)$. In the present study, we demonstrate, in vivo, similar imprinting effects of $\mathrm{GH}$, to determine the earliest observable age when the imprinting effects are expressed, and now identify those CYP2C11 required transcription factors affected by GH imprinting. In addition, we have measured mRNA and/or protein levels of male-specific CYP3A2, femalepredominant CYP2C6 as well as signal transducer and activator of transcription 5 (STAT5), Janus kinase 2 (JAK2), suppressors of cytokine signaling (SOCS1, SOCS2, CIS), hepatocyte nuclear factor 6 (HNF6) and B-cell lymphoma 6 (Bcl6); factors potentially involved in GH imprinting of CYP expression.

\section{Materials and methods}

\section{Animal experimentation}

Rats were housed in the University of Pennsylvania Laboratory Animal Resources facility under the supervision of certified laboratory animal medicine veterinarians and were treated according to the research protocol approved by the Institutional Animal Care and Use Committee of the University. Housing conditions, as well as breeding and treatment protocols, were reported previously $(22,23)$. Basically, neonatal male rat pups (Crl:CD(sd)BR) were injected, s.c., with either of two of four possible treatments. (i) The potent and permanent GH blocker $(20,23)$ monosodium glutamate (MSG), $4 \mathrm{mg} / \mathrm{g}$ body weight on alternate days, starting within $24 \mathrm{~h}$ of birth for a total of five injections. (ii) An equivalent amount of MSG NaCI diluent. (iii) Recombinant rat GH ( $2 \mathrm{IU} / \mathrm{mg}), 20 \mu \mathrm{g} / 100 \mathrm{~g}$ body weight, twice/day for the first 12 days of life. (iv) An equivalent amount of the GH bicarbonate diluent. Accordingly, control pups received treatments 2 and 4; MSG pups received treatments 1 and 4; MSG+GH pups received treatments 1 and 3 . At 28 days of age, the recently weaned male rats were injected, i.p., with either rat GH $(20 \mu \mathrm{g} / 100$ g body weight) twice per day for 5 days or an equivalent amount of GH diluent. The rats were euthanized $30 \mathrm{~min}$ following the tenth injection at 32 days of age. GH was purchased from Dr A F Parlow, National Hormone and Peptide Program, Harbor-UCLA Medical Center, Torrance, CA. 


\section{RNA isolation}

Total RNA from liver tissue was isolated using Trizol reagent (Life Technologies), purified with Qiagen RNeasy mini kit, and treated with DNase I in order to remove any traces of genomic DNA using RNase-Free DNaseSet (Qiagen) according to the manufacturer's protocol. RNA concentrations and purity were determined by UV spectrophotometry (A260/280 > 1.8 and A260/240 > 1.7) and integrity was verified by the intensities of $28 \mathrm{~S}$ and $18 \mathrm{~S}$ rRNA bands on a denaturing agarose gel visualized on a FluorChem IS-8800 Imager (Alpha Innotech, San Leandro, CA).

\section{Quantitative RT-PCR}

Cyp2C11, Cyp2C6, Jak2, Cis, Socs1, Socs2, Hnf6, and Bcl6 gene expressions were determined by quantitative RT-PCR (qRT-PCR) using SYBR green on an Applied Biosystem StepOnePlus Real-Time PCR System (Life Technologies). RNA isolation, concentration, and purity determination were performed as mentioned previously. cDNA synthesis was completed using the High-Capacity RNA-to-cDNA kit (Life Technologies) as per instructions with appropriate no-RT (-RT) and non-template controls. PCR primers for Cyp2C11 and Cyp2C6 (24), Cis, Socs1 and Socs2 (21) Jak2 (25), Hnf6 (26), and Bcl6 (27) were synthesized by Integrated DNA Technologies (IDT, Coralville, IA). Gene expression was determined using peptidylprolyl isomerase A, Ppia (28), one of only a few housekeeping genes unaffected by sex and GH (29).

\section{Western blotting}

Whole cell lysate protein (30) was assayed for individual CYP isoforms and transcription factors by Western blotting (31). Briefly, $50 \mu \mathrm{g}$ of protein were electrophoresed on $1.00 \mathrm{~mm}$-thick SDS-polyacrylamide (10-12\%) gels and electroblotted onto nitrocellulose membranes. The blots were probed with monoclonal anti-rat CYP2C11 and antirat CYP3A2 (Detroit R \& D Inc., Detroit, MI), monoclonal anti-rat CYP2C6 and polyclonal anti-SOCS2 (Santa Cruz Biotechnology, Inc.), monoclonal anti-pSTAT5 and polyclonal anti-pJAK2 (Cell Signaling Technology) and polyclonal anti-JAK2 (Upstate Cell Signaling Solutions) and detected with an enhanced chemiluminescence kit (Amersham, GE Healthcare Bio-Sciences Corp). Signals were normalized to a known control sample, for example, pSTAT5, pJAK2, etc., repeatedly run on all blots and/or to the expression of $\beta$-actin (Sigma-Aldrich). The protein signals were scanned and the densitometric units were obtained as integrated density values quantitated using a FluorChem IS-8800 Imager (Alpha Innotech) software supplied with the gel documentation system.

\section{Statistical analysis}

Puberty is a major life event during which profound anatomic, biochemical, physiologic, and behavioral systematic changes occur, of which sex-dependent parameters, such as we measured, are particularly responsive. Since each animal enters puberty at a somewhat different age, it was not surprising that a few rats were at an extreme place on the puberty spectrum at 32 days of age so that we occasionally came across an obvious statistical outliers (32) and, accordingly, they were eliminated from further statistical analyses. Otherwise, all data were subjected to ANOVA, and differences were determined using $t$ statistics and the Bonferroni procedure for multiple comparisons.

\section{Results}

\section{Developmental GH irreversibly programs hepatic CYP2C11 responsiveness to episodic $\mathrm{GH}$ regulation}

CYP2C11 is the principal male-dependent form, comprising $>50 \%$ of the total CYP isoforms in adult male rat liver (19), but effectively undetectable in the female liver, explaining its designation as male-specific (15). Furthermore, CYP2C11 expression is first observed during puberty $(33,34)$ as a result of the inductive effects of a concomitant pubertyinitiated secretion of the masculine episodic GH profile $(35,36)$. In this regard, we chose to examine the earliest possible effects of neonatal GH imprinting on CYP2C11 expression. Consequently, at 32 days of age, around the earliest outset of male puberty as characterized by the start of spermatogenesis, some of the control rats were just beginning to express small, above prepubertal levels of CYP2C11, whereas other rats were far more developmentally advanced; some approaching adult-like levels of the isoform (Fig. 1). Since puberty occurs during a relatively prolonged developmental period, it is not surprising that individual animals would exhibit pubertal changes (e.g. CYP2C11 expression) at different rates.

Expression of episodic GH-dependent CYP2C11 was near totally suppressed in the liver from GH-devoid, neonatally MSG-treated male rats (Fig. 1). In contrast, neonatal administration of both MSG and GH resulted in substantial expression levels of CYP2C11 ( 30\% of control values) in the developmentally precocious cohort;

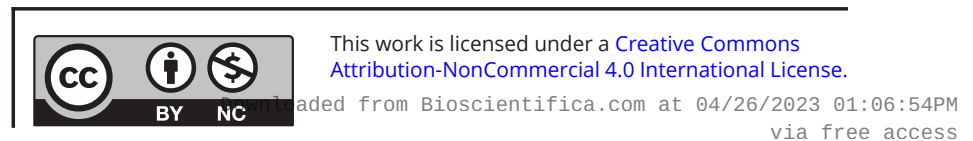



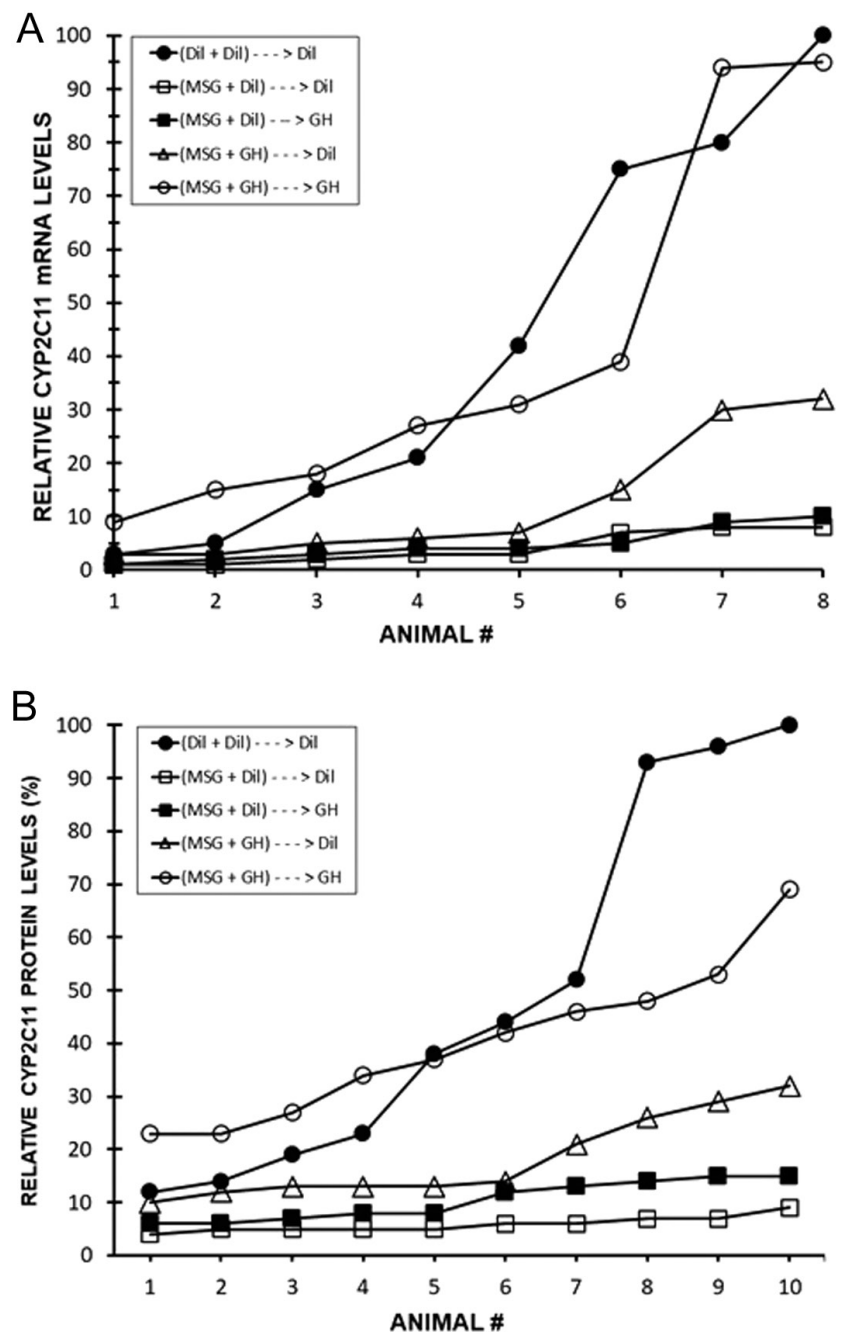

Figure 1

Hepatic male-specific CYP2C11 mRNA (A) and protein (B) levels in peripubertal (32 days old) male rats challenged with rat $\mathrm{GH}$ and previously treated as neonates with rat GH and/or MSG. Isoform levels are presented as individual values per animal so that increasing animal numbers are assigned to increasing CYP2C11 concentrations. Neonatal treatments are indicated in parentheses while peripubertal treatments are shown following the broken arrows. See 'Materials and methods' section for all treatment details.

this in spite of the same total absence of circulating $\mathrm{GH}$ as observed in the MSG-treated rats. (Neonatal and subsequent adult circulating $\mathrm{GH}$ profiles resulting from all the present treatments have been reported elsewhere $(6$, $21,23)$.) In this regard, a minimal baseline level of hepatic CYP2C11 ( 20 to $40 \%$ of normal) can be expressed in adults in the absence of $\mathrm{GH}$, whereas full induction of the isoform requires exposure to the masculine episodic $\mathrm{GH}$ profile $(36,37,38)$. Consequently, these findings indicate that neonatal GH programs developmental mechanisms responsible for the expression of GH-independent, baseline CYP2C11 expression.
Five days of exposure (i.e. 28-32 days of age) to an episodic GH profile (i.e. two i.p. injections/day) were completely ineffective in inducing CYP2C11 expression in the MSG-alone treated neonates resulting in a permanent refractory response to the hormone. In contrast, peripubertal males neonatally treated with both MSG and GH responded to the episodic GH injections with a similar increase in CYP2C11 mRNA as observed in normal control rats (Fig. 1A). Protein levels of the isoform followed a similar inductive trajectory as controls, but never quite expressed the same maximum concentrations (Fig. 1B), possibly as a result of the mode of GH administration. That is, the most effective method of restoring normal CYP2C11 levels to GH-devoid rats is to renaturalize the physiologic circulating GH profiles of 6 daily pulses at $\sim 1 \mathrm{~h}$ in duration, each interrupted by an interpulse absence of plasma GH for $\sim 2 \mathrm{~h}$ duration by administering the hormone via an indwelling right atrial catheter attached to an electronic pulse simulator (38, 39). Since the rats in the present study were too small to tolerate the procedure, and s.c. injections fail miserably to replicate the physiologic GH profile (40), we chose to administer two i.p. injections of rat $\mathrm{GH}$ whose absorption would mimic i.v. administration. In agreement with our present findings, we have reported $(20,39)$ that two daily i.v. pulses of rat GH to hypophysectomized (HYPOX) rats completely restored CYP2C11 mRNA concentrations, whereas protein levels and catalytic activity of the isoform increased to only $~ 65$ to $70 \%$ of normal; a similar finding observed with other CYPs treated with suboptimal doses of inducers (41).

\section{Developmental GH permanently imprints CYP2C6 suppression by episodic GH}

Although titulary female-predominant, the sex difference in CYP2C6 is minimal compared to CYP2C11. Moreover, unlike CYP2C11, GH suppresses CYP2C6 expression (the masculine episodic profile more so than the feminine continuous profile), so that adult HYPOX maximizes the expression of the isoform eliminating sex differences (38, $42,43)$. In agreement, peripubertal male rats neonatally treated with MSG (i.e. MSG-alone or MSG+GH) and subsequently injected with GH-vehicle, like the HYPOX rat, expressed maximal concentrations of CYP2C6 that were considerably greater than intact controls (Fig. 2A). Administration of episodic GH to these GH-devoid rats reduced CYP2C6 mRNA and protein levels only in the males treated neonatally with both $\mathrm{MSG}+\mathrm{GH}$, demonstrating that the developmental hormone irreversibly imprints

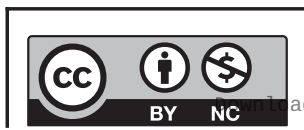

This work is licensed under a Creative Commons Attribution-NonCommercial 4.0 International License. ded from Bioscientifica.com at 04/26/2023 01:06:54PM 
GH suppression of CYP2C6 in male rats. (Recall that the HYPOX rat is normally imprinted by perinatal GH and like CYP2C6 in the neonatally MSG+GH treated rats, responds to the suppressive effects of restored episodic GH treatment $(38,42,43))$. In agreement, additional statistical comparisons $(P<0.01)$ of CYP2C6 levels (mRNA and protein) between male rats that received either diluent or GH as neonates, but otherwise both treated neonatally with MSG and peripubertal GH (Fig. 2) demonstrated the suppressive imprinting effects of neonatal GH in response to peripubertal GH.

\section{$\mathrm{GH}$ regulation of CYP3A2 expression is programmed by developmental $\mathrm{GH}$}

Unlike male-specific CYP2C11 whose expression is dependent on the initiation of puberty, adult-like expression levels of male-specific CYP3A2 have been observed as early as the first and second week of life (33, 34). The different developmental patterns may be due to the fact that adult levels of CYP2C11 are dependent on the secretion of the masculine episodic GH profile at puberty, whereas maximum CYP3A2 levels are expressed in the absence of GH (e.g. HYPOX); the episodic profile being somewhat repressive, whereas the feminine profile is entirely suppressive $(38,44)$.

We observed that neonatal exposure to MSG-alone resulted in a small but significant repression of CYP3A2 protein that responded to an inductive effect of episodic GH administration (Fig. 2B). The CYP3A2 findings in the MSG-alone treated males were reverse of that observed in HYPOX male rats $(38,44,45,46)$. Whereas both animal models are examples of GH depletion, CYP3A2 is repressed in the MSG rats, but over-expressed in the HYPOX rats. Moreover, administration of the episodic GH profile induced CYP3A2 in the MSG rats but suppressed the isoform in the HYPOX rats. A relevant difference between these animals is imprinting; the MSG-treated rats are not imprinted by GH, whereas the adult HYPOX rats are normal perinates developmentally exposed to endogenous GH. These findings would suggest that early GH imprints CYP3A2 expression. In agreement, when the neonates were exposed to MSG as well as GH, they expressed normal control-like levels of CYP3A2 (Fig. 2B). Unfortunately, unlike normally GH-imprinted rats, for example, adult HYPOX (39), two daily GH pulses were unable to alter CYP3A2 levels in the neonatal MSG + GH perinates. In this regard, CYP3A2, in contrast to other CYPs, only responds (in this case suppression) to the fully restored physiologic circulating GH profile of 6 daily pulses each containing
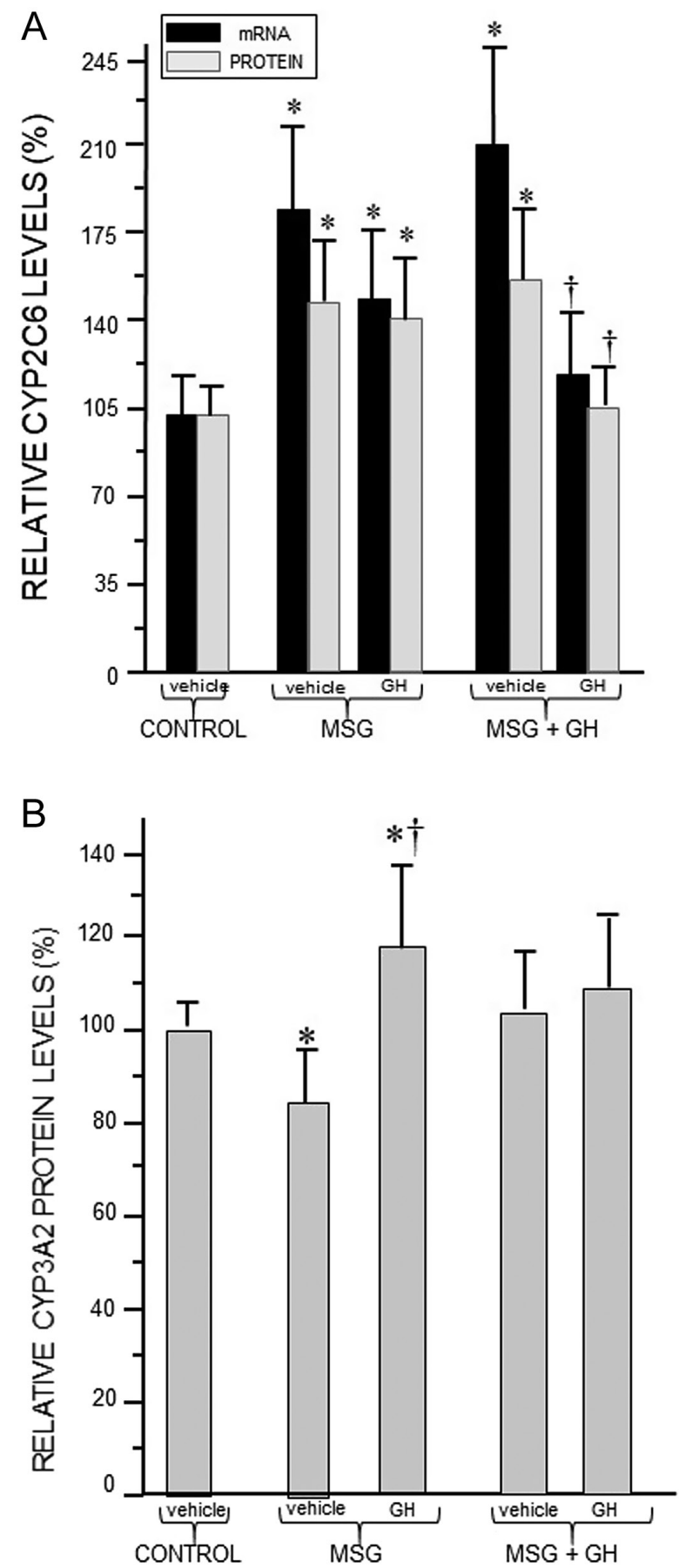

Figure 2

Hepatic female-predominant CYP2C6 mRNA and protein levels (A) and male-specific CYP3A2 protein levels (B) in peripubertal (32 days old) male rats challenged with rat $\mathrm{GH}$ and previously treated as neonates with rat $\mathrm{GH}$ and/or MSG. Peripubertal treatments are indicated within the brackets along the $\mathrm{x}$-axis while the neonatal treatments, in a larger font, are below the brackets. Results are presented as a percentage of mRNA and protein in control rats arbitrarily designated as $100 \%$. Values are means \pm s.D. of at least eight rats/data point. $* P<0.01$ (CYP2C6) or $* P<$ 0.05(CYP3A2), vs CONTROL; ${ }^{\dagger} P<0.01$ compares peripubertal GH treatment with vehicle treatment of rats exposed to the same neonatal treatment. See 'Materials and methods' section for all treatment details. 
$>3.5 \mu \mathrm{g} \mathrm{GH} / \mathrm{min} / \mathrm{mL}$ plasma $(38,39,47)$. An additional statistical comparison found no difference in CYP3A2 when comparing the isoform in rats that received either GH diluent or GH as neonates, but otherwise, both groups were treated neonatally with MSG and peripubertal GH. As just mentioned, CYP3A2 in GH-imprinted males, including HYPOX, is only responsive to the normal GH secretory profile. Accordingly, our CYP3A2 conclusions have to be based on the results of rats exposed only to neonatal MSG.

\section{Episodic GH activation of STAT5 is dependent on developmental GH imprinting}

The terminal transducer in the signaling transduction pathway mediating episodic GH induction of CYP2C11 is STAT5b. This transcription factor must be activated (i.e. phosphorylated), homodimerized, and translocated to the nucleus, where it binds to a promoter site initiating transcription of the Cyp2C11 gene (48). The livers from all animals in every treatment group were analyzed 30 min after the last GH pulse; an optimal time to measure the STAT5b response (49). In this study, we used an antibody against pSTAT5 (i.e. the activated form) that might recognize pSTAT5a as well as pSTAT5b. However, the male liver contains considerably less STAT5a than STAT5b, and only the latter is obligatory for the expression of the male pattern of hepatic gene expression $(48,50)$. Furthermore, whereas the masculine episodic GH profile can activate both forms of STAT5, activation levels of $5 \mathrm{~b}$ are substantially greater than $5 \mathrm{a}$ (49).

Peripubertal challenge with GH caused less than a doubling in pSTAT5 concentrations in the MSG-alone treated rats, but a 12-fold increase in the neonates treated with both MSG and GH (Fig. 3), indicating that maximal, that is, effective (49), activation of STAT5, likely STAT5b, by episodic GH requires perinatal GH imprinting. In this regard, GH activation of STAT5b is generally commensurate with nuclear translocation and subsequent binding to the Cyp2C11 promoter $(6,21)$. The fact that peripubertal GH was capable of activating STAT5 in animals that did not express CYP2C11 (Fig. 1) agrees with previous findings that $\mathrm{GH}$ activation of the transcription factor developmentally precedes the ability of the liver to express CYP2C11 $(50,51)$. Moreover, we found that the large variation in pSTAT5 induction levels did not statistically correlate with the variations in individual CYP2C11 levels, revealing the normal, though highly variable, ontogenetic expression profile of the transcription factor.

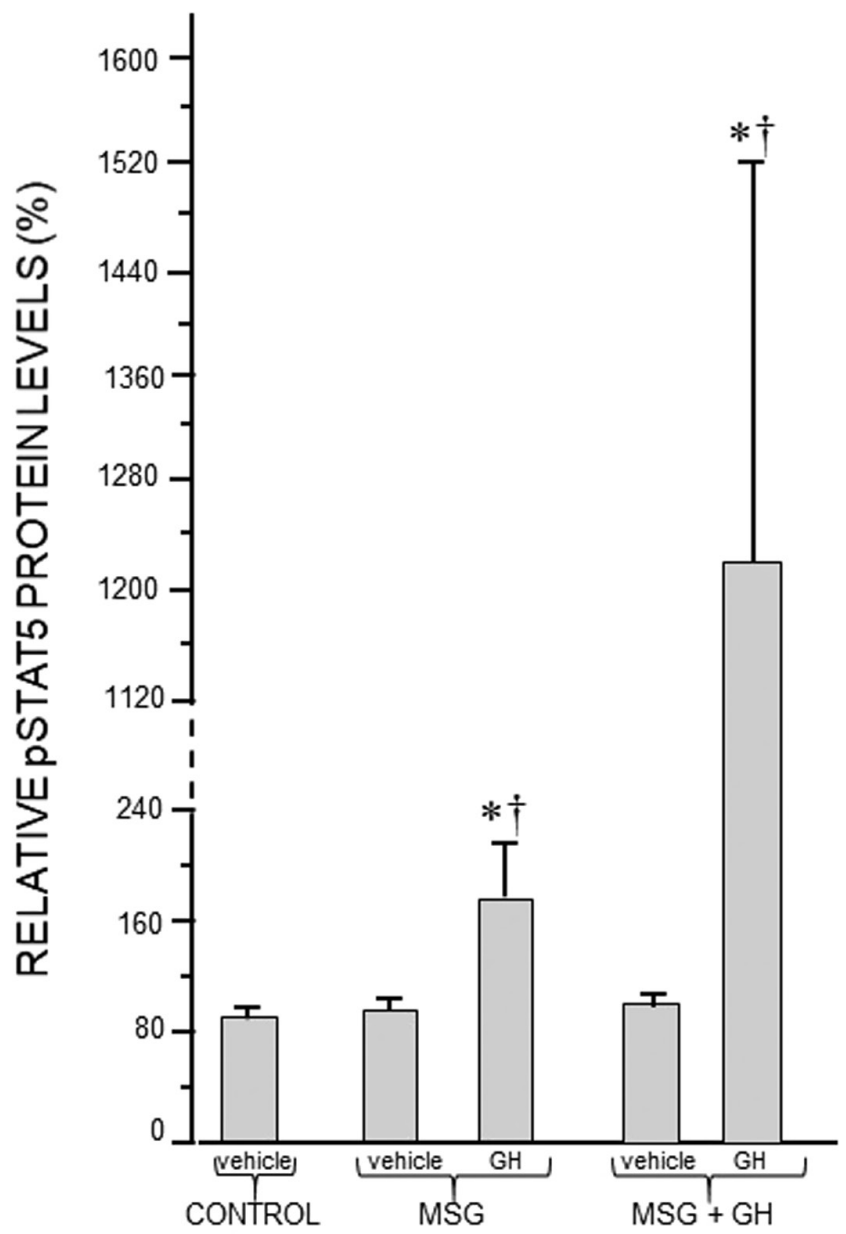

Figure 3

Hepatic PSTAT5 protein levels in peripubertal ( 32 days old) male rats challenged with rat $\mathrm{GH}$ and previously treated as neonates with rat $\mathrm{GH}$ and/or MSG. Peripubertal treatments are indicated within the brackets along the $x$-axis while the neonatal treatments, in a larger font, are below the brackets. Results are presented as a percentage of protein in control rats arbitrarily designated as $100 \%$. Values are means \pm S.D. of at least eight rats/data point. ${ }^{*} P<0.01$, vs control; ${ }^{\dagger} P<0.01$ compares peripubertal GH treatment with vehicle treatment of rats exposed to the same neonatal treatment. See 'Materials and methods' section for all treatment details. Note the break in the y-axis.

\section{Regulation of JAK2 is not dependent on GH imprinting}

Activation of JAK2 by the hormone-bound growth hormone receptor (GHR) is an early step in the JAK2STAT5b-CYP2C11 pathway $(18,48,50)$. Neonatal exposure to either MSG or MSG + GH resulted in elevated JAK2 mRNA and protein levels that were about 2.5 to 1.5 times, respectively, greater than control concentrations (Fig. 4). When the two MSG groups were peripubertally treated with $\mathrm{GH}$, JAK2 levels declined, more so in the MSG+GH group, to control-like concentrations. Due to a paucity of material, we were limited to measuring pJAK2 in only two or three

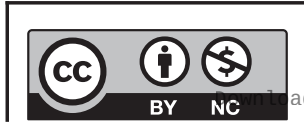

This work is licensed under a Creative Commons Attribution-NonCommercial 4.0 International License. ded from Bioscientifica.com at 04/26/2023 01:06:54PM 
mRNA PROTEIN

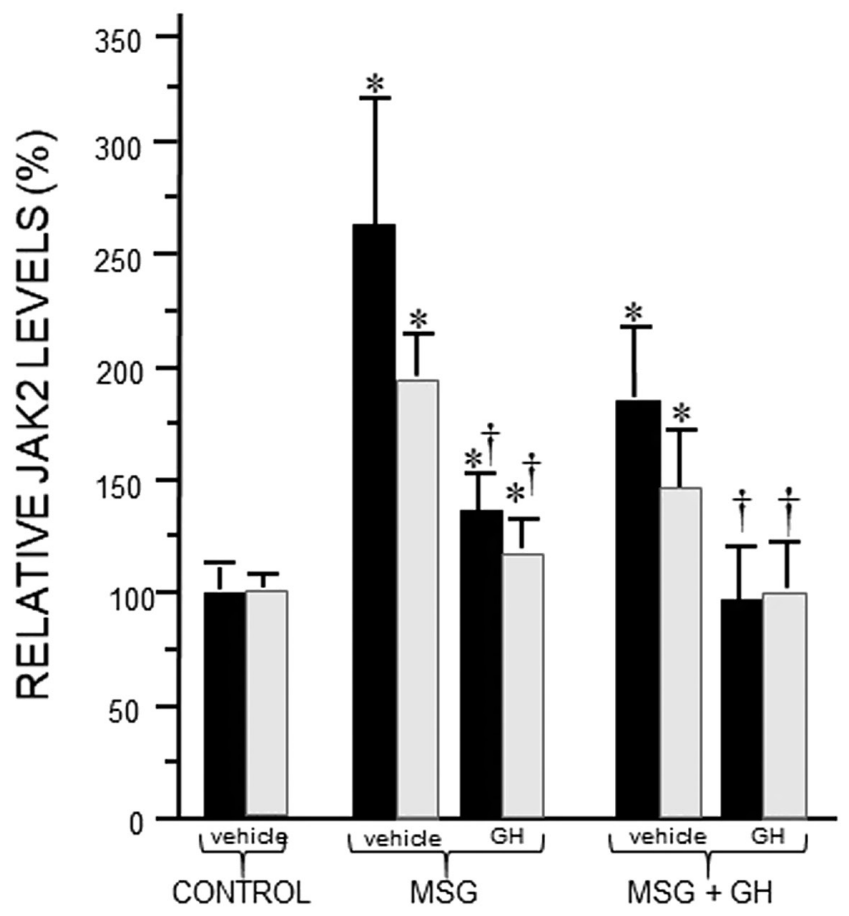

Figure 4

Hepatic JAK2 mRNA and protein levels in peripubertal (32 days old) male rats challenged with rat $\mathrm{GH}$ and previously treated as neonates with rat GH and/or MSG. Peripubertal treatments are indicated within the brackets along the $\mathrm{x}$-axis while the neonatal treatments, in a larger font, are below the brackets. Results are presented as a percentage of mRNA and protein in control rats arbitrarily designated as $100 \%$. Values are means \pm S.D. of at least eight rats/data point. ${ }^{*} P<0.01$, vs control; ${ }^{\dagger} P<$ 0.01 compares peripubertal GH treatment with vehicle treatment of rats exposed to the same neonatal treatment. See 'Materials and methods' section for all treatment details.

animals from the peripubertal GH injected groups (not presented). Although found at much lower concentrations, pJAK2 levels appeared to reflect that of total JAK2. Although pJAK2 has been reported to increase rapidly following GH administration, it declines to near baseline after $\sim 30 \mathrm{~min}$ $(18,49)$ as we observed with JAK2 (Fig. 4). Nevertheless, other than a somewhat elevated response in the MSGalone groups, we found nothing that would suggest GH imprinting of JAK2 regulation. Since JAK2 mediates the actions of many non-GH factors (52), it seems reasonable that its expression would not depend on GH imprinting.

\section{Developmental GH imprints optimal response of the SOCS family of inhibitory proteins to episodic $\mathrm{GH}$ regulation}

The SOCS family of inhibitory proteins are negative regulators of GH signaling. Their actions are particularly important in regulating the effects of episodic GH on such male-dependent parameters as growth, musculoskeletal metabolism, and CYP expression $(18,48)$. Consequently, we decided to examine the early effects of neonatal GH on both the baseline levels (i.e. sans GH) and the responsiveness to peripubertal $\mathrm{GH}$ of three of the most commonly referenced SOCS, that is, CIS, SOCS1, and SOCS2. (We had previously reported that SOCS3 was neither imprinted by GH (21) nor responsive to the actions of episodic GH (18). Consequently, we omitted analysis of SOCS3 from the present study.)

Earlier, we $(18,21)$, as well as others (48) had implicated SOCS2 as a requisite factor in the regulation of the episodic GH-GHR-JAK2-STAT5b-CYP2C11 pathway. SOCS2 mRNA in both the neonatally MSG and MSG + GH treated males exhibited the same highly depressed concentrations (Fig. 5) as observed in GH-depleted HYPOX rats $(18,27,53)$. When the animals were challenged, peripubertally, with episodic $\mathrm{GH}, \mathrm{mRNA}$ levels doubled in the MSG-alone treatment group but quadrupled in the MSG + GH group. Protein levels of SOCS2 were much less responsive to GH than the mRNA. In fact, only the MSG $+\mathrm{GH}$ treatment group exhibited a small increase in SOCS2 protein following peripubertal GH injections. This finding is quite understandable in light of previous reports demonstrating that transcription levels of the SOCS family may be detectable $30 \mathrm{~min}$ following a GH pulse (the time of our study) but peaks after 90 min (18, $27,53)$. Consequently, translation of the SOCS proteins lags considerably behind transcription initiation which, in fact, is the required timing for terminating GHR-JAK2 activation of STAT5b (48). The present finding supports the conclusion that developmental GH is required, at least, for optimal induction of SOCS2 by episodic GH.

CIS, another member of the SOCS family of inhibitory proteins, has also been implicated in the regulation of the episodic GH-GHR-JAK2-STAT5b-CYP2C11 pathway (18, 21). Similar to HYPOX rats (53), CIS mRNA levels in the MSG-alone treated males were $~ 35 \%$ lower than controls but rebounded to control-like concentrations following episodic GH administration (Fig. 6A). In contrast, rats neonatally exposed to MSG and GH exhibited the same level of CIS mRNA as controls that were also normally exposed to neonatal $\mathrm{GH}$. When the $\mathrm{MSG}+\mathrm{GH}$ rats were peripubertally challenged with episodic $\mathrm{GH}$, like normal animals $(18,52)$, they responded with a near six-fold increase in levels of the transcript. Thus, we see that exposure to developmental $\mathrm{GH}$ is required for episodic GH induction of CIS mRNA.

Lastly, we measured the effects of developmental GH on the peripubertal expression of SOCS1 mRNA (Fig. 6B). In agreement with SOCS2 (Fig. 5) and CIS (Fig. 6A) findings,

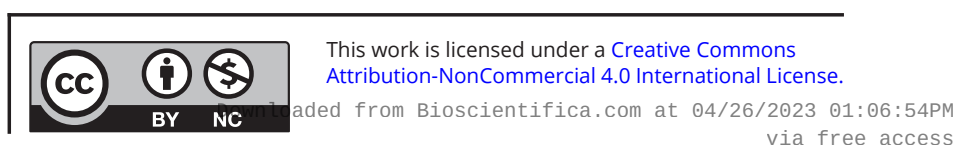




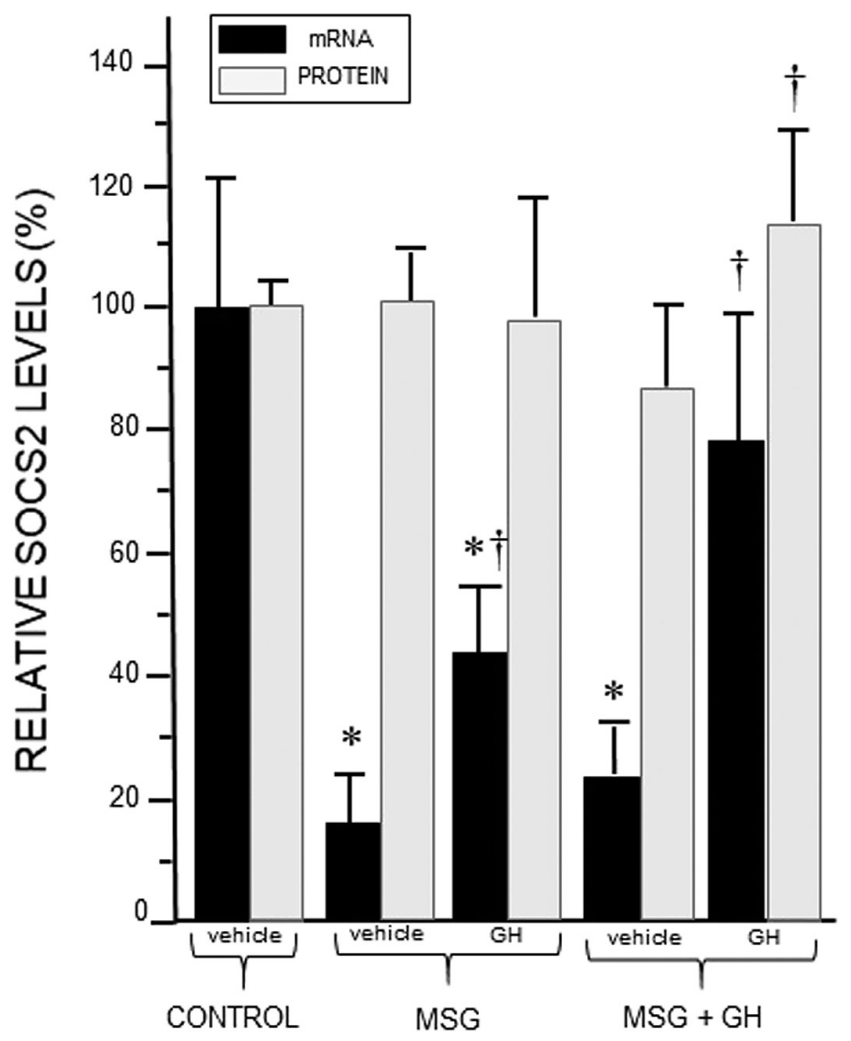

Figure 5

Hepatic SOCS2 mRNA and protein levels in peripubertal (32 days old) male rats challenged with rat $\mathrm{GH}$ and previously treated as neonates with rat GH and/or MSG. Peripubertal treatments are indicated within the brackets along the $\mathrm{x}$-axis while the neonatal treatments, in a larger font, are below the brackets. Results are presented as a percentage of mRNA and protein in control rats arbitrarily designated as $100 \%$. Values are means \pm S.D. of at least eight rats/data point. ${ }^{*} P<0.01$, vs control; ${ }^{\dagger} P<$ 0.01 compares peripubertal GH treatment with vehicle treatment of rats exposed to the same neonatal treatment. See 'Materials and methods' section for all treatment details.

SOCS1 levels were reduced in both the GH-depleted MSG and MSG + GH rats, more so in the latter. Whereas episodic GH injections resulted in a significant decline in SOCS1 mRNA concentrations in the MSG-alone treated males, neonatal exposure to GH (imprinting) blocked the effects of MSG, responding to peripubertal GH with a two-fold increase in transcript levels.

\section{Regulation of HNF6 is not dependent on GH imprinting}

The liver-enriched transcription factor HNF6 is more highly expressed in female than male liver and serves to transactivate the female-specific CYP2C12 promoter. The continuous secretory $\mathrm{GH}$ profile characteristic of the female rat increases HNF6 expression, which in synergy with HNF3 stimulates CYP2C12 expression while simultaneously
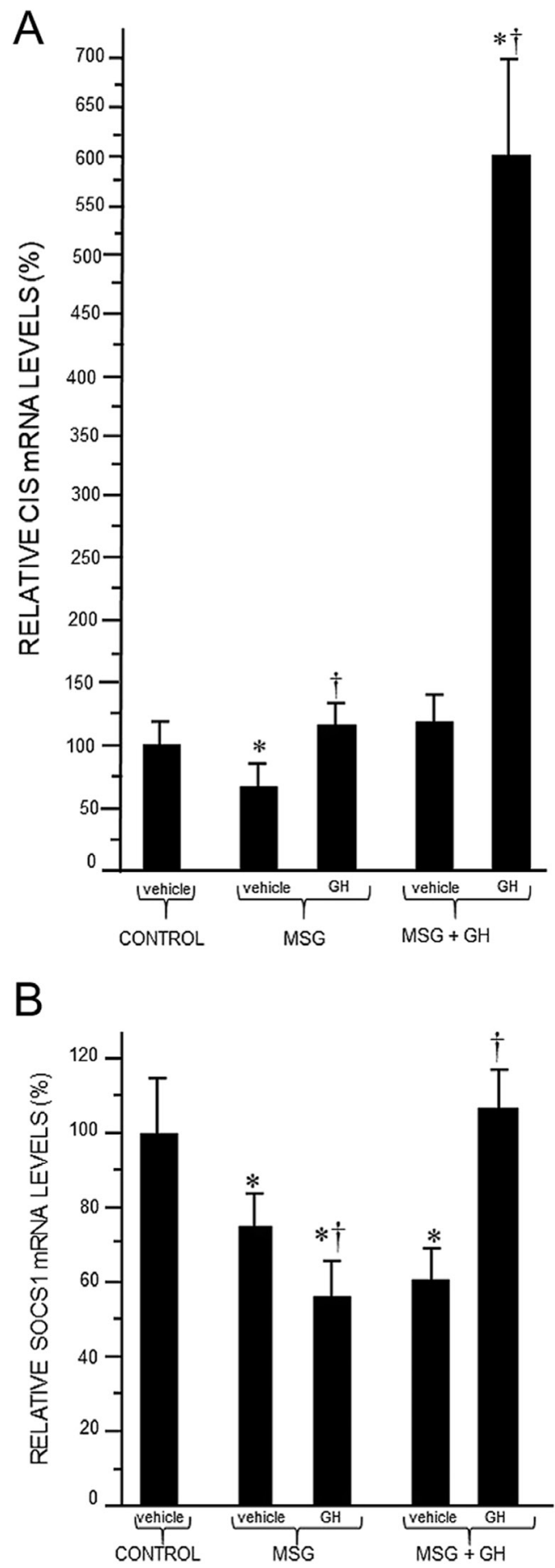

Figure 6

Hepatic CIS (A) and SOCS1 (B) mRNA levels in peripubertal (32 days old) male rats challenged with rat $\mathrm{GH}$ and previously treated as neonates with rat GH and/or MSG. Peripubertal treatments are indicated within the brackets along the $\mathrm{x}$-axis while the neonatal treatments, in a larger font, are below the brackets. Results are presented as a percentage of mRNA in control rats arbitrarily designated as $100 \%$. Values are means \pm S.D. of at least eight rats/data point. ${ }^{*} P<0.01$, vs control; ${ }^{\dagger} P<0.01$ compares peripubertal GH treatment with vehicle treatment of rats exposed to the same neonatal treatment. See 'Materials and methods' section for all treatment details. 


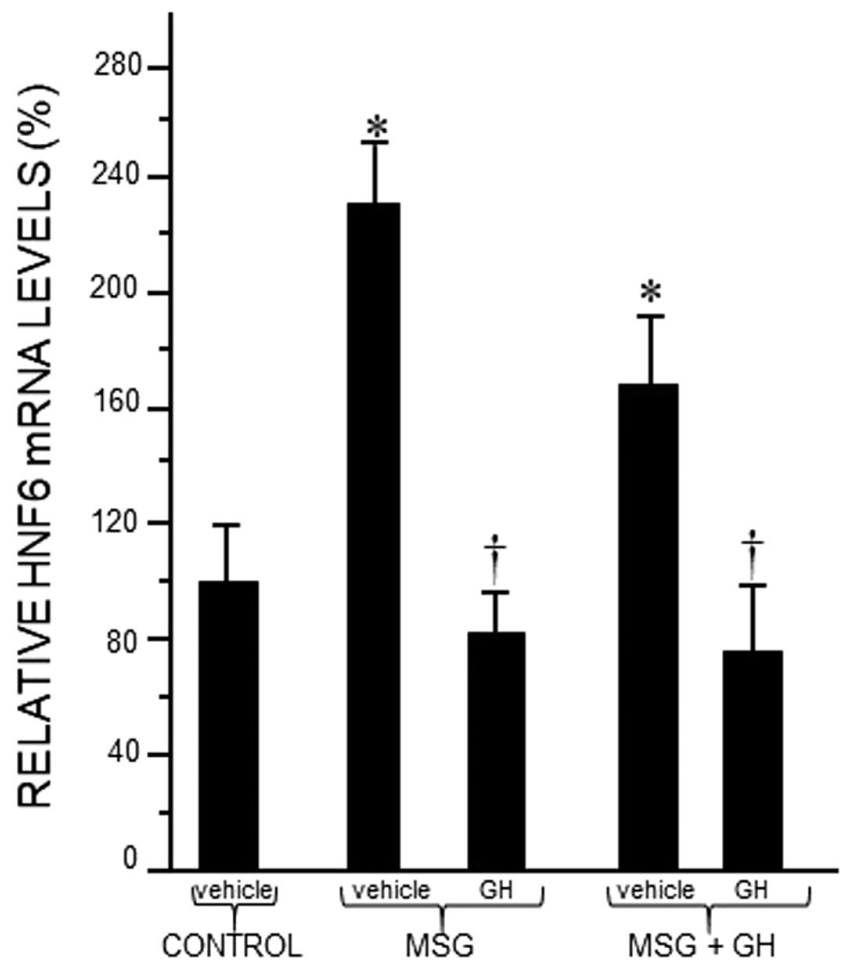

Figure 7

Hepatic HNF6 mRNA levels in peripubertal (32 days old) male rats challenged with rat $\mathrm{GH}$ and previously treated as neonates with rat $\mathrm{GH}$ and/or MSG. Peripubertal treatments are indicated within the brackets along the $\mathrm{x}$-axis while the neonatal treatments, in a larger font, are below the brackets. Results are presented as a percentage of mRNA in control rats arbitrarily designated as $100 \%$. Values are means \pm S.D. of at least eight rats/data point. ${ }^{*} P<0.01$, vs control; ${ }^{\dagger} P<0.01$ compares peripubertal GH treatment with vehicle treatment of rats exposed to the same neonatal treatment. See 'Materials and methods' section for all treatment details.

repressing male-specific CYP2C11. In contrast, the masculine episodic GH profile activates hepatic STAT5b, which blocks HNF6, as well as HNF3 expression, which in turn prevents CYP2C12 induction and allows for CYP2C11 expression $(54,55)$. In agreement, we have found that episodic GH injections reduced HNF6 mRNA concentrations several folds in both MSG-alone and MSG+GH treated males (Fig. 7) suggesting that regulation of the transcription factor in male rats does not require GH imprinting. This conclusion seems reasonable considering that HNF6 is basically a female-dependent factor required for the expression of female-specific CYP2C12 and there would appear to be no reason for its expression to be imprinted in males.

\section{Developmental GH imprints Bcl6 expression}

The transcriptional repressor $\mathrm{Bcl} 6$ is a male-specific rat hepatic gene protein elevated following HYPOX, suppressed by pulses of $\mathrm{GH}$, and implicated in murine

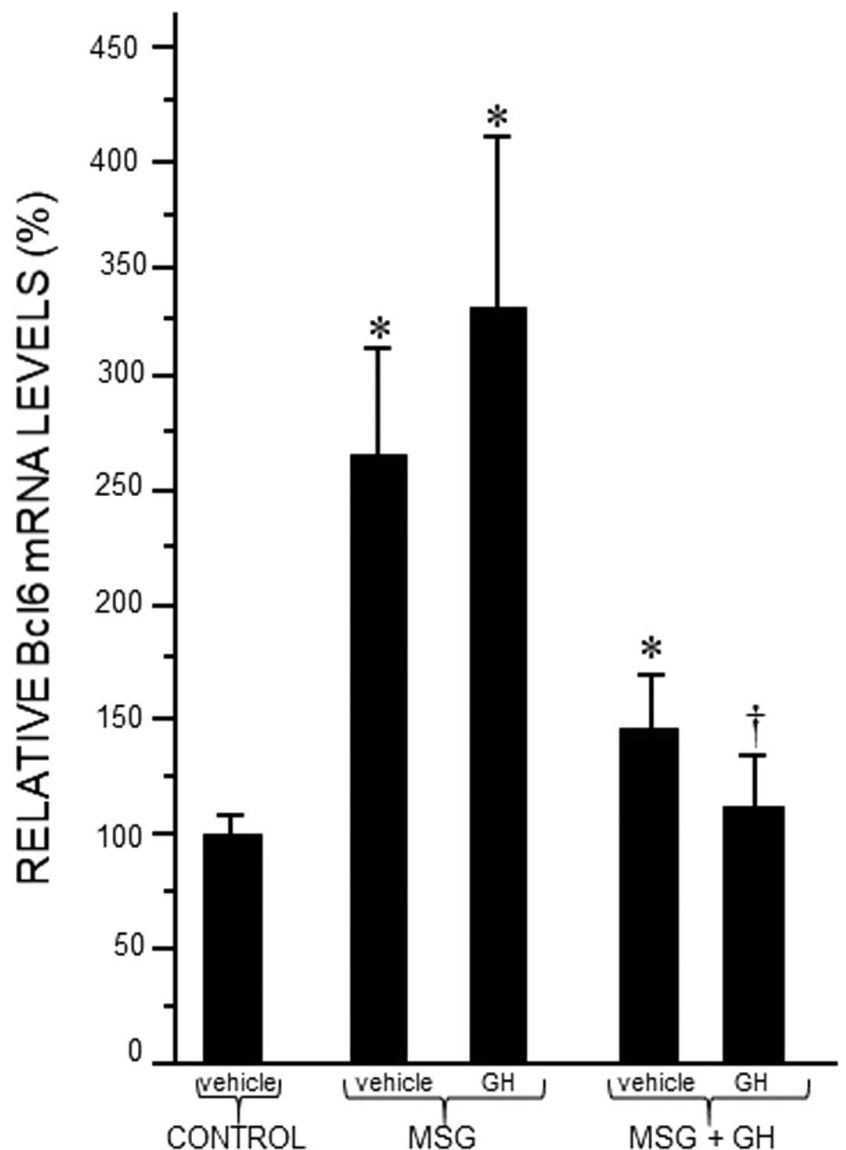

Figure 8

Hepatic Bcl6 mRNA levels in peripubertal (32 days old) male rats challenged with rat $\mathrm{GH}$ and previously treated as neonates with rat $\mathrm{GH}$ and/or MSG. Peripubertal treatments are indicated within the brackets along the $\mathrm{x}$-axis while the neonatal treatments, in a larger font, are below the brackets. Results are presented as a percentage of mRNA in control rats arbitrarily designated as $100 \%$. Values are means \pm S.D. of at least eight rats/data point. ${ }^{*} P<0.01$, vs control; ${ }^{\dagger} P<0.01$ compares peripubertal GH treatment with vehicle treatment of rats exposed to the same neonatal treatment. See 'Materials and methods' section for all treatment details.

sex-dependent CYP expression $(27,56)$. In agreement, we observed that GH-devoid MSG-alone and MSG+GH peripubertal males expressed elevated concentrations of Bcl6 mRNA, more so in the former (Fig. 8). When the rats were challenged with episodic GH injections, Bcl6 mRNA levels were unresponsive and remained elevated in the MSG-alone treated males but were suppressed to controllike levels in the MSG+GH group. These results indicate that normal expression levels of Bcl6 mRNA in both the presence and absence of peripubertal GH are dependent on developmental GH. However, due to a paucity of information regarding the role of Bcl6 in the regulatory expression of male-specific CYPs in the rat, any significance of our finding could only be speculative.

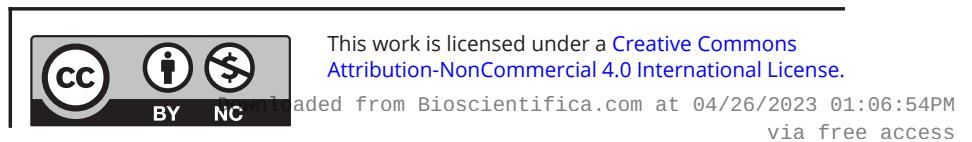




\section{Discussion}

The adult responsiveness to a particular hormone is predetermined, that is, imprinted, during development by this same hormone $(7,57)$, and in agreement, we have reported that regulation of adult drug metabolism by GH is accordingly imprinted by postpartum GH (6, 12). To examine the developmental process by which perinatal GH imprints adult CYP expression, we blocked GH secretion in newborn male rat pups with MSG, a specific disrupter of the differentiating hypothalamic GH-releasing hormone nuclei $(58,59)$, while having no effect on liver development (45). Furthermore, a cohort of MSG-treated neonates were simultaneously administered physiologic replacement levels of rat GH during this 'critical (i.e. irreversible) developmental period' for the hypothalamic-pituitary-hepatic axis $(19,21)$. Whereas as little as the first dose of neonatal MSG nearly eliminates $\mathrm{GH}$ from the circulation, concurrent $\mathrm{GH}$ replacement at the specified dosage and length of treatment restores the normal age-dependent plasma concentrations of the hormone in affected pups (21). However, following the withdrawal of GH treatment, plasma GH concentrations become undetectable in both MSG-treated groups, those receiving and those not receiving neonatal GH replacement, as the hypothalamic GH regulatory nuclei were permanently destroyed by MSG in both groups $(6,21)$. Regardless of the same total absence of plasma $\mathrm{GH}$, the two MSG treated groups expressed antithetic peripubertal expression levels of hepatic CYP2C11. The MSG-alone treated males exhibited a total loss of CYP2C11 mRNA and protein. In contrast, the neonatally MSG + GH exposed rats, also having no detectable plasma levels of GH, expressed $30 \%$ control levels of CYP2C11. Evidently, even though there was no circulating GH after 12 days of age in the MSG + GH treatment group, neonatal replacement $\mathrm{GH}$ alone appears to have programmed a permanent liver imprint allowing for GH-independent baseline expression of CYP2C11 not observed in the MSGalone treatment group. Moreover, peripubertal males not imprinted by neonatal GH (MSG only treatment) were completely unresponsive to episodic GH induction of CYP2C11 expression. In contrast, when the same neonatal treatment was supplemented with rat $\mathrm{GH}$, the rats were responsive to the episodic GH profile exhibiting a dramatic induction of CYP2C11, establishing perinatal $\mathrm{GH}$ as a requisite developmental organizer or imprinter, in an in vivo model.

In addition to examining the effects of GH imprinting on CYP2C11 expression, we also included, in the present study, male-specific CYP3A2 and female-predominant CYP2C6. In contrast to male-specific CYP2C11 whose optimal levels are dependent on the presence of the masculine episodic GH profile, maximum CYP3A2 levels are expressed in the absence of $\mathrm{GH}$; the episodic $\mathrm{GH}$ profile being somewhat repressive $(38,44)$. CYP3A2 expression in animals not imprinted by GH (i.e. MSG-alone) exhibited the complete opposite response to GH than that of normal controls. That is, in the absence of circulating GH, CYP3A2 concentrations declined but rebounded above normal when exposed to episodic GH. In contrast, CYP3A2 concentrations in the MSG + GH rats did not decline in the absence of circulating GH nor did episodic GH produce an elevation of the isoform. These findings suggest that developmental GH imprints the CYP3A2 response; whether in the postpubertal absence or presence of the hormone.

CYP2C6 expression levels are repressed by $\mathrm{GH}$ hormone; more so by the masculine episodic profile than the feminine continuous profile, explaining its designation as female-predominant $(38,42,43)$. In agreement, peripubertal male rats neonatally treated with MSG (i.e. MSG-alone or MSG $+\mathrm{GH}$ ) expressed CYP2C6 concentrations that were considerably greater than intact controls. However, administration of episodic GH reduced CYP2C6 levels only in the animals treated neonatally with both $\mathrm{MSG}+\mathrm{GH}$, demonstrating that the normal suppressive effects of episodic GH are dependent on imprinting by developmental GH.

The present findings indicate that CYP2C11 is not the only isoform in male rats imprinted by developmental GH. Whereas the imprinting effects of developmental GH are most dramatically demonstrated by CYP2C11, the more subtle, though still physiologically significant effects on CYP3A2 and CYP2C6 may be explained by the fact that, unlike CYP2C11, neither CYP3A2 nor CYP2C6 is dependent on GH for their expression and consequently, may be less dependent on its imprinting. Moreover, episodic GH acts as an inducer, but instead, acts as a repressor in regulating CYP3A2 and CYP2C6 expression. Similarly, delimited effects of GH imprinting on CYP isoforms expressed in female rats have been reported (60).

What, then, are the defects in the GH-inductive response of CYP2C11, the isoform for which we have the most mechanistic information, in adult rats neonatally treated with MSG? That is, why is the isoform unable to respond to GH? In this regard, GH signaling in the liver by the episodic profile (in contrast to the continuous profile) is initiated by hormone binding to and the resulting activation of the GHR on the surface of the target cell. This allows for the recruitment and/or activation of two 
molecules of JAK2, which then cross-phosphorylate each other, as well as phosphorylating the receptor on key tyrosine residues. STAT5b, a latent transcription factor, binds to these phosphorylated receptor docking sites and is, in turn, phosphorylated and homodimerizes and translocates to the nucleus, where it binds to promoter sites initiating transcription of GH-regulated genes. An important negative regulatory mechanism of $\mathrm{GH}$ signaling, the SOCS family of inhibitory proteins, which, depending on the protein, inhibit GH signaling by inactivating JAK2, by competing with STAT5b for common tyrosine-binding sites on the intracellular tail of the GHR, and/or by the proteasome-dependent mechanism that results in the degradation of the (GH-GHR-Jak2)-SOCS complex $(48,61,62)$. At least four possible components in this signal transduction pathway as well as the associated transcription repressor Bcl6 were permanently altered by neonatal MSG. The response to episodic GH of neonatally $\mathrm{MSG}+\mathrm{GH}$ treated rats increased hepatic CIS, SOCS1, SOCS2, as well as pSTAT5 by $\sim 6-, \sim 2-, \sim 4-$, and $\sim 12$-fold, respectively. With the exception of SOCS1 whose levels were reduced by episodic GH; CIS, SOCS2, and pSTAT5 concentrations were increased by an average of $\sim 2$-fold in the MSG-alone treated perinates. Here we see the greatest difference in findings between the present in vivo study with that of previous reports using primary hepatocyte cultures $(6,21)$ that found CIS, SOCS1, SOCS2, and PSTAT5b to be totally unresponsive to episodic GH in hepatocytes from MSG-alone treated male rats. Unlike the hepatocyte cultures, however, the livers from the in vivo study were exposed to factors, that is, cytokines, other than GH that could have been responsible for the small induction levels of the affected transducers in the MSG-alone treated male rats. Clearly, then, neonatal exposure to MSG irreversibly suppresses GH activation of CYP2C11-dependent signal transduction at multiple steps. Whether the multiple defects in the signaling pathway are all independently induced or rather the result of an initial upstream defect (i.e. GHR) cascading dysfunctions down the signaling pathway requires further investigation.

The considerable peripubertal variation in CYP2C11 expression levels ranging from barely detectable to nearadult concentrations in the GH-imprinted rats compared to the activation of all measured dependent signaling factors suggests that the signal transduction pathways required for GH induction of CYP2C11 are in place before the developmental expression of the isoform.

Our findings demonstrate that perinatal GH imprints a sexually dimorphic, latently expressed program, which at its earliest expression, is subsequently responsive to
GH during puberty. The imprinting is obligatory and occurs during a critical developmental period, whereas the imprinted program is first expressed around the time of puberty. Since there is a myriad of metabolic functions in humans, including growth rates and lean body mass; cardiovascular, bone, adipose, and muscle physiology; protein, carbohydrate, lipid, and electrolyte metabolism all regulated, at least in part, by GH, and like CYP expression, exhibit permanent sexual dimorphisms (cf. ref 1), it may be that these adult functions also require perinatal GH imprinting and could be vulnerable to pediatric drug therapy known to inadvertently disrupt GH secretion.

\section{Declaration of interest}

The authors declare that there is no conflict of interest that could be perceived as prejudicing the impartiality of the research report.

\section{Funding}

This work was supported by the U.S. National Institutes of Health, Eunice Kennedy Shriver National Institute of Child Health and Human Development (grant HD-061285) and principally, the Chutzpah Foundation (\#112942).

\section{Author contribution statement}

B H S designed the study with input from S B and A M H. S B and A M $\mathrm{H}$ performed the actual experiments. $\mathrm{B} \mathrm{H} \mathrm{S}$ analyzed the data with suggestions from S B and A M H. B H S wrote the manuscript approved by $\mathrm{SB}$ and $\mathrm{AM} \mathrm{H}$.

\section{References}

1 Shapiro BH, Agrawal AK \& Pampori NA. Gender differences in drug metabolism regulated by growth hormone. International Journal of Biochemistry and Cell Biology 199527 9-20. (https://doi. org/10.1016/1357-2725(94)00056-5)

2 Waxman DJ \& Holloway MG. Sex differences in the expression of hepatic drug metabolizing enzymes. Molecular Pharmacology 200976 215-228. (https://doi.org/10.1124/mol.109.056705)

3 Thangavel C, Boopathi E \& Shapiro BH. Intrinsic sexually dimorphic expression of the principal human CYP3A4 correlated with suboptimal activation of GH/glucocorticoid-dependent transcriptional pathways in men. Endocrinology 2011152 4813-4824. (https://doi.org/10.1210/en.2011-1274)

4 Thangavel C, Boopathi E \& Shapiro BH. Inherent sex-dependent regulation of human hepatic CYP3A5. British Journal of Pharmacology 2013168 988-1000. (https://doi.org/10.1111/j.14765381.2012.02222.x)

5 Legraverend C, Mode A, Wells T, Robinson I \& Gustafsson JA. Hepatic steroid hydroxylating enzymes are controlled by the sexually dimorphic pattern of growth hormone secretion in normal and dwarf rats. FASEB Journal 19926 711-718. (https://doi.org/10.1096/ fasebj.6.2.1537461)

6 Das RK, Banerjee S \& Shapiro BH. Irreversible perinatal imprinting of adult expression of the principal sex-dependent drug-metabolizing 
enzyme CYP2C11. FASEB Journal 201428 4111-4122. (https://doi. org/10.1096/fj.13-248864)

7 Csaba G. Hormonal imprinting: phylogeny, ontogeny, diseases and possible role in present-day human evolution. Cell Biochemistry and Function 200826 1-10. (https://doi.org/10.1002/cbf.1412)

8 Goldman AS. Animal models of inborn errors of steroidogenesis and steroid action. In Mammalian Reproduction, Colloquium der Gesellschaft für Biologische Chemie, pp. 9-11. Eds H Gibian \& EJ Plotz. Berlin: Springer, 1970.

9 Feder NH. Perinatal hormones and their role in the development of sexually dimorphic behaviors. In Neuroendocrinology of Reproduction, pp. 127-158. Ed NT Adler. New York: Plenum Press, 1987.

10 Shapiro BH. A paradox in development: masculinization of the brain without androgen receptors. Progress in Clinical and Biological Research 1985171 151-173.

11 Shapiro BH, Levine DC \& Adler NT. The testicular feminized rat: a naturally occurring model of androgen independent brain masculinization. Science 1980209 418-420. (https://doi.org/10.1126/ science.7384816)

12 Pampori NA \& Shapiro BH. Gender differences in the responsiveness of the sex-dependent isoforms of hepatic P450 to the feminine plasma growth hormone profile. Endocrinology 1999140 1245-1254. (https:// doi.org/10.1210/endo.140.3.6545)

13 Thangavel C, Garcia MC \& Shapiro BH. Intrinsic sex differences determine expression of growth hormone-regulated female cytochrome P450s. Molecular and Cellular Endocrinology 2004220 31-39. (https://doi.org/10.1016/j.mce.2004.04.002)

14 Dhir RN, Dworakowski W, Thangavel C \& Shapiro BH. Sexually dimorphic regulation of hepatic isoforms of human cytochrome P450 by growth hormone. Journal of Pharmacology and Experimental Therapeutics 2006316 87-94. (https://doi.org/10.1124/jpet.105.093773)

15 Thangavel C \& Shapiro BH. Inherent sexually dimorphic expression of hepatic CYP2C12 correlated with repressed activation of growth hormone-regulated signal transduction in male rats. Drug Metabolism and Disposition 200836 1884-1895. (https://doi.org/10.1124/ dmd.108.021451)

16 Thangavel C, Dworakowski W \& Shapiro BH. Inducibility of malespecific isoforms of cytochrome $\mathrm{P} 450$ by sex-dependent growth hormone profiles in hepatocyte cultures from male but not female rats. Drug Metabolism and Disposition 200634 410-419. (https://doi. org/10.1124/dmd.105.007716)

17 Dhir RN, Thangavel C \& Shapiro BH. Attenuated expression of episodic growth hormone-induced CYP2C11 in female rats associated with suboptimal activation of the Jak2/Stat5B and other modulating signaling pathways. Drug Metabolism and Disposition 200735 2102-2110. (https://doi.org/10.1124/dmd.107.017475)

18 Thangavel C \& Shapiro BH. A molecular basis for the sexually dimorphic response to growth hormone. Endocrinology 2007148 2894-2903. (https://doi.org/10.1210/en.2006-1333)

19 Morgan ET, MacGeoch C \& Gustafsson JA. Hormonal and developmental regulation of expression of the hepatic microsomal steroid 16 alpha-hydroxylase cytochrome P-450 apoprotein in the rat. Journal of Biological Chemistry 1985260 11895-11898. (https://doi. org/10.1016/S0021-9258(17)38963-9)

20 Shapiro BH, Pampori NA, Ram PA \& Waxman DJ. Irreversible suppression of growth hormone-dependent cytochrome P450 2C11 in adult rats neonatally treated with monosodium glutamate. Journal of Pharmacology and Experimental Therapeutics 1993265 979-984.

21 Das RK, Banerjee S \& Shapiro BH. Growth hormone: a newly identified developmental organizer. Journal of Endocrinology 2017232 377-389. (https://doi.org/10.1530/JOE-16-0471)

22 Shapiro BH, MacLeod JN, Pampori NA, Morrissey JJ, Lapenson DP \& Waxman DJ. Signaling elements in the ultradian rhythm of circulating growth hormone regulating expression of sex-dependent forms of hepatic cytochrome P450. Endocrinology 1989125 2935-2944. (https://doi.org/10.1210/endo-125-6-2935)
23 Pampori NA, Agrawal AK, Waxman DJ \& Shapiro BH. Differential effects of neonatally administered glutamate on the ultradian pattern of circulating growth hormone regulating expression of sexdependent forms of cytochrome P450. Biochemical Pharmacology 1991 41 1299-1309. (https://doi.org/10.1016/0006-2952(91)90101-a)

24 Das RK, Banerjee S \& Shapiro BH. Noncanonical suppression of GH-dependent isoforms of cytochrome $\mathrm{P} 450$ by the somatostatin analog octreotide. Journal of Endocrinology 2013216 87-97. (https:// doi.org/10.1530/JOE-12-0255)

25 Sutherland JM, Frost ER, Ford EA, Peters AE, Reed NL, Seldon AN, Mihalas BP, Russel DL, Dunning KR \& McLaughlin EA. Janus kinase JAK1 maintains the ovarian reserve of primordial follicles in the mouse ovary. Molecular Human Reproduction 201824 533-542. (https:// doi.org/10.1093/molehr/gay041)

26 Falix FA, Weeda VB, Labruyere WT, Poncy A, de Waart DR, Hakvoort TBM, Lemaigre F, Gaemers IC, Aronson DC \& Lamers WH. Hepatic Notch2 deficiency leads to bile duct agenesis perinatally and secondary bile duct formation after weaning. Developmental Biology 2014396 201-213. (https://doi.org/10.1016/j.ydbio.2014.10.002)

27 Meyer RD, Laz EV, Su T \& Waxman DJ. Male-specific hepatic Bcl6: growth hormone-induced block of transcription elongation in females and binding to target genes inversely coordinated with STAT5. Molecular Endocrinology 200923 1914-1926. (https://doi.org/10.1210/ me.2009-0242)

28 Feroze-Merzoug F, Berquin IM, Dey J \& Chen YQ. Peptidylprolyl isomerase A (PPIA) as a preferred internal control over GAPDH and beta-actin in quantitative RNA analyses. BioTechniques 200232 776-782. (https://doi.org/10.2144/02324st03)

29 Das RK, Banerjee S \& Shapiro BH. Extensive sex- and/or hormonedependent expression of rat housekeeping genes. Endocrine Research 201338 105-111. (https://doi.org/10.3109/07435800.2012.723294)

30 Garcia MC, Thangavel C \& Shapiro BH. Epidermal growth factor regulation of female-dependent CYP2A1 and CYP2C12 in primary rat hepatocyte culture. Drug Metabolism and Disposition 200129 111-120.

31 Pampori NA, Pampori MK \& Shapiro BH. Dilution of the chemiluminescence reagents reduces the background noise on western blots. BioTechniques 199518 588-590.

32 Grubbs FE. Procedures for detecting outlying observations in samples. Technometrics 196911 1-21. (https://doi.org/10.1080/00401706.1969.1 0490657)

33 Waxman DJ, Dannan GA \& Guengerich FP. Regulation of rat hepatic cytochrome P-450: age-dependent expression, hormonal imprinting, and xenobiotic inducibility of sex-specific isoenzymes. Biochemistry 198524 4409-4417. (https://doi.org/10.1021/bi00337a023)

34 Agrawal AK \& Shapiro BH. Latent overexpression of hepatic CYP2C7 in adult male and female rats neonatally exposed to phenobarbital: a developmental profile of gender-dependent P450s. Journal of Pharmacology and Experimental Therapeutics 2000293 1027-1033.

35 Edén S. Age- and sex-related differences in episodic growth hormone secretion in the rat. Endocrinology 1979105 555-560. (https://doi. org/10.1210/endo-105-2-555)

36 Gabriel SM, Roncancio JR \& Ruiz NS. Growth hormone pulsatility and the endocrine milieu during sexual maturation in male and female rats. Neuroendocrinology 199256 619-625. (https://doi. org/10.1159/000126284)

37 Waxman DJ, Pampori NA, Ram PA, Agrawal AK \& Shapiro BH. Interpulse interval in circulating growth hormone patterns regulates sexually dimorphic expression of hepatic cytochrome P450. PNAS 199188 6868-6872. (https://doi.org/10.1073/pnas.88.15.6868)

38 Agrawal AK \& Shapiro BH. Differential expression of genderdependent hepatic isoforms of cytochrome $\mathrm{P}-450$ by pulse signals in the circulating masculine episodic growth hormone profile of the rat. Journal of Pharmacology and Experimental Therapeutics 2000292 228-237.

39 Agrawal AK \& Shapiro BH. Intrinsic signals in the sexually dimorphic circulating growth hormone profiles of the rat. Molecular and Cellular

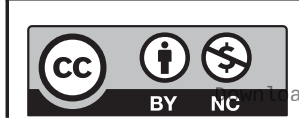

This work is licensed under a Creative Commons Attribution-NonCommercial 4.0 International License. ded from Bioscientifica com at 04/26/2023 01:06:54PM 
Endocrinology 2001173 167-181. (https://doi.org/10.1016/s03037207(00)00401-9)

40 Pampori NA, Agrawal AK \& Shapiro BH. Renaturalizing the sexually dimorphic profiles of circulating growth hormone in hypophysectomized rats. Acta Endocrinologica 1991124 283-289. (https://doi.org/10.1530/acta.0.1240283)

41 Agrawal AK \& Shapiro BH. Phenobarbital induction of hepatic CYP2B1 and CYP2B2: pre- and post-transcriptional effects of gender, adult age, and phenobarbital dose. Molecular Pharmacology 199649 523-531.

42 Westin S, Ström A, Gustafsson JA \& Zaphiropoulos PG. Growth hormone regulation of the cytochrome P-450IIC subfamily in the rat: inductive, repressive, and transcriptional effects on P-450f (IIC7) and P-450PB1 (IIC6) gene expression. Molecular Pharmacology 199038 192-197.

43 Pampori NA \& Shapiro BH. Feminization of hepatic cytochrome P450s by nominal levels of growth hormone in the feminine plasma profile. Molecular Pharmacology 199650 1148-1156.

44 Waxman DJ, LeBlanc GA, Morrissey JJ, Staunton J \& Lapenson DP. Adult male-specific and neonatally programmed rat hepatic P-450 forms RLM2 and 2a are not dependent on pulsatile plasma growth hormone for expression. Journal of Biological Chemistry 1988263 11396-11406. (https://doi.org/10.1016/S0021-9258(18)37970-5)

45 Kaufhold A, Nigam PK, Dhir RN \& Shapiro BH. Prevention of latently expressed CYP2C11, CYP3A2, and growth hormone defects in neonatally monosodium glutamate-treated male rats by the $\mathrm{N}$-methyl-D-aspartate receptor antagonist dizocilpine maleate. Journal of Pharmacology and Experimental Therapeutics 2002302 490-496. (https://doi.org/10.1124/jpet.102.034785)

46 Waxman DJ, Ram PA, Pampori NA \& Shapiro BH. Growth hormone regulation of male-specific rat liver P450s $2 \mathrm{~A} 2$ and $3 \mathrm{~A} 2$ : induction by intermittent growth hormone pulses in male but not female rats rendered growth hormone deficient by neonatal monosodium glutamate. Molecular Pharmacology 199548 790-797.

47 Agrawal AK, Pampori NA \& Shapiro BH. Neonatal phenobarbitalinduced defects in age- and sex-specific growth hormone profiles regulating monooxygenases. American Journal of Physiology 1995268 E439-E445. (https://doi.org/10.1152/ajpendo.1995.268.3.E439)

48 Waxman DJ \& Frank SJ. Growth hormone action. In Principles of Molecular Regulation, pp. 55-83. Eds PM Conn \& AR Means. Totowa: Humana Press, 2000

49 Verma AS, Dhir RN \& Shapiro BH. Inadequacy of the Janus kinase 2/ signal transducer and activator of transcription signal transduction pathway to mediate episodic growth hormone-dependent regulation of hepatic CYP2C11. Molecular Pharmacology 200567 891-901. (https://doi.org/10.1124/mol.104.005454)

50 Choi HK \& Waxman DJ. Plasma growth hormone pulse activation of hepatic JAK-STAT5 signaling: developmental regulation and role in male-specific liver gene expression. Endocrinology 2000141 3245-3255. (https://doi.org/10.1210/endo.141.9.7638)
51 Phornphutkul C, Frick GP, Goodman HM, Berry SA \& Gruppuso PA. Hepatic growth hormone signaling in the late gestation fetal rat. Endocrinology 2000141 3527-3533. (https://doi.org/10.1210/ endo.141.10.7702)

52 Roskoski R. Janus kinase (JAK) inhibitors in the treatment of inflammatory and neoplastic diseases. Pharmacological Research 2016 111 784-803. (https://doi.org/10.1016/j.phrs.2016.07.038)

53 Tollet-Egnell P, Flores-Morales A, Stavréus-Evers A, Sahlin L \& Norstedt G. Growth hormone regulation of SOCS-2, SOCS-3, and CIS messenger ribonucleic acid expression in the rat. Endocrinology 1999 140 3693-3704. (https://doi.org/10.1210/endo.140.8.6878)

54 Lahuna O, Fernandez L, Karisson H, Maiter D, Lemaigre FP, Rousseau GG, Gustafsson J \& Mode A. Expression of hepatocyte nuclear factor 6 in rat liver is sex-dependent and regulated by growth hormone. PNAS 199794 12309-12313. (https://doi.org/10.1073/ pnas.94.23.12309)

55 Delesque-Touchard N, Park SH \& Waxman DJ. Synergistic action of hepatocyte nuclear factors 3 and 6 on CYP2C12 gene expression and suppression by growth hormone-activated STAT5b: proposed model for female specific expression of CYP2C12 in adult rat liver. Journal of Biological Chemistry 2000275 34173-34182. (https://doi.org/10.1074/ jbc.M004027200)

56 Shapiro BH \& Goldman AS. New thoughts on sexual differentiation of the brain. In Genetic Mechanisms of Sexual Development, pp. 221-251. Eds HL Vallet \& IH Porter. New York: Academic Press, 1979.

57 Chikada H, Ida K, Ando E, Inagaki Y, Sakamoto A \& Kamiya A. Establishment and analysis of a mouse model that regulates sexrelated differences in liver drug metabolism. Journal of Technical Methods and Pathology 201898 1500-1511. (https://doi.org/10.1038/ s41374-018-0088-6)

58 Bakke JL, Lawrence N, Bennett J, Robinson S \& Bowers CY. Late endocrine effects of administering monosodium glutamate to neonatal rats. Neuroendocrinology 197826 220-228. (https://doi. org/10.1159/000122829)

59 Dada MO, Campbell GT \& Blake CA. Effects of neonatal administration of monosodium glutamate on somatotrophs and growth hormone secretion in prepubertal male and female rats. Endocrinology 1984115 996-1003. (https://doi.org/10.1210/endo-1153-996)

60 Banerjee S, Das RK \& Shapiro BH. Feminization imprinted by developmental growth hormone. Molecular and Cellular Endocrinology 2019479 27-38. (https://doi.org/10.1016/j. mce.2018.08.011)

61 Karlsson H, Gustafsson JA \& Mode A. Cis desensitizes GH induced Stat5 signaling in rat liver cells. Molecular and Cellular Endocrinology 1999154 37-43. (https://doi.org/10.1016/s0303-7207(99)00101-x)

62 Leroith D \& Nissley P. Knock your SOCS off! Journal of Clinical Investigation 2005115 233-236. (https://doi.org/10.1172/JCI24228)

Received in final form 25 June 2021

Accepted 20 August 2021

Accepted Manuscript published online 23 August 2021 https://ec.bioscientifica.com https://doi.org/10.1530/EC-21-0143 (c) 2021 The authors Published by Bioscientifica Ltd

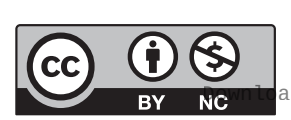

This work is licensed under a Creative Commons Attribution-NonCommercial 4.0 International License. ded from Bioscientifica.com at 04/26/2023 01:06:54PM 